\title{
Developing Digital Control System Centrifugal Pumping Unit
}

\author{
Digital Control Systems
}

\author{
Safarini Osama \\ IT Department \\ University of Tabuk, \\ Tabuk, KSA
}

\begin{abstract}
Currently, the leading Russian oil companies engaged in the development of automatic control systems (ACS) oil equipment, to improve its efficiency.

As an example of an object needed to optimize its work, a centrifugal pumping unit (CPU) can be specified. Frequency actuators provide an opportunity to run CPU in any algorithm, and many industrial controllers, are part of the automatic control system technological process (ACSTP), allow optimizing the operation of the centrifugal pumping unit and help determine the requirements for the development of digital automatic control system for CPU.

The development of digital automatic control systems for such a complex nonlinear control object like CPU has several features that should be considered when designing control systems for that object.
\end{abstract}

Keywords- ACS - Automatic Control System; CPU-Centrifugal Pumping Unit; PID-A proportional-integral-derivative.

\section{INTRODUCTION}

Modern status of oil producing companies is characterized by an increase in water content well product, which leads to increased costs of oil production.

One possibility to reducing costs is to use optimization methods of field equipment, through the development and implementation of optimal digital automatic control systems oilfield objects.

The solution of this problem consists of several stages and involves the development of models of control objects (primarily nonlinear) for the synthesis of regulators, for the simulation of control systems on digital computers, as well as developing methods for designing digital control systems and means to optimize their work.

\section{DISCUSSION}

In the synthesis of ACS CPU must consider several system requirements, determined by the characteristics of the controlled object and features of its operation. One such requirement is the frequency of the time discretization (sampling), which is mainly determined by the response time to emergencies. Frequency of issuing solutions of ACS is governed by RD-35.240.00-KTN-207-08 JSC AK "Transneft", according to which the processing of signals in the controller does not exceed $0.1 \mathrm{~s}$. Thus, the frequency of issuing control actions cannot be less than $10 \mathrm{~Hz}$.

The second system requirement is a record of the presence of noise and interference in the measured values of management technological parameters. In practice, the pumping units are often located far away from the controller, which leads to significant levels of noise and interference in the measured values of adjustable parameters. A third feature to be considered in the design of ACS CPU is the presence of delays in information channels. Time delays of measured parameters and control signals lead to a significant reduction in the reserves stability of ACS CPU.

The next feature to be considered when designing the ACS CPU, The presence of an integral component of the control signal to the most widely used PID control algorithm [1] [2]. In the short-term disruptions in the supply voltage, important integral component of the PID can lead to disaster. It is therefore necessary to minimize the possibility of an integral component in the control signal and, furthermore, restricts its maximum value.

The fifth is particularly true nonlinear CPU as the object of control, making it necessary to change the controller parameters depending on the mode of operation.

As a parameter characterizing the mode of operation, for example, is chosen the rotation frequency of induction motor drive. To eliminate the problems associated with a step-change in the coefficients of the regulator [3], it is useful to determine their functionality depending on the mode of operation, for example, in the form of polynomials.

At the final stage of the development of ACS CPU, it is necessary to analyze the stability of the system. Low stability margins lead to instability and breakdown of equipment and armature CPU. Sustainable control system must meet engineering standards of quality, such as $\Delta \varphi \geq 45^{\circ}, \Delta L \geq$ $12 \mathrm{~dB}$ where $\Delta \varphi$ and $\Delta L$ - system stability margins in phase and amplitude, respectively [4].

\section{DESIGNING ACS CPU BASED ON SYSTEM FEATURES}


As an example, the control system designed to meet the above functioning features, can lead the pressure of ACS to the output of CPU [5]:

$$
\begin{aligned}
& \delta W_{i}=\left(P_{i}^{p r}-P_{i}\right)-K_{D} B_{1, i} \cdot\left(P_{i}-P_{i-1}\right)+B_{2, i} \cdot\left(\varphi_{i}-\right. \\
& \left.\varphi_{i-1}\right) \text {, } \\
& \delta W_{\text {Int }, i}=\delta W_{I n t, i-1}+\delta W_{i} \cdot \tau, \\
& \delta W_{i}=K_{I} \cdot \delta W_{I n t, i}+K_{P} \cdot \delta W_{i}^{p} \text {, }
\end{aligned}
$$

Where - $\boldsymbol{\delta} \boldsymbol{W}_{\boldsymbol{i}}$ power, supplied to the engine in the curren cycle control;

$P_{i}^{p r}$ - Relative value of a given fluid pressure in the i-th step of control;

$\boldsymbol{P}_{\boldsymbol{i}}, \boldsymbol{P}_{\boldsymbol{i}-\mathbf{1}}$ - The relative fluid pressure on the current and previous steps of control;

$\boldsymbol{\varphi}_{i}, \boldsymbol{\varphi}_{i-1}$ - The relative frequency of rotation of the CPU drive for current and previous steps of control;

\section{$\boldsymbol{\tau}$ - Duration of one cycle control.}

The numerical values of the parameters $\boldsymbol{B}_{\mathbf{1}, \boldsymbol{i}}$ and $\boldsymbol{B}_{\mathbf{2}, \boldsymbol{i}}$ are determined in the process of design of ACS CPU.

The coefficients $\mathrm{K}_{P}, \mathrm{~K}_{D}$ and $\mathrm{K}_{I}$ are the coefficients of proportionality, differentiation and integration, respectively.

The value of the integration step ACS was chosen equal to the $\boldsymbol{\tau}=0.025 \mathrm{sec}$. Thus, the frequency output of control influence ACS is $40 \mathrm{~Hz}$.

In this case, reception, signal processing and issuance of the control action took three steps of control or 0.075 seconds, which satisfies the requirements of the regulations in 0.1 seconds.

The proposed implementation scheme ACS CPU (1) is based on the PID Act and is distinguished by the presence of additional feedback from the derivative rotation frequency of the drive of CPU.

The advantage of this structure is that it allows taking into account the dynamics of the drive motor and, thereby increasing the stability margins of the control system of fluid pressure.

To minimize the value of the integral component in the total control signal is necessary to obtain maximum transfer coefficient of the system while keeping the monotonic nature of the transition processes.

To do this, it is necessary to find the optimal value of the coefficients $\mathrm{K}_{P}, \mathrm{~K}_{D}$ and $\mathrm{K}_{I}$ by the method presented in [6]. The technique allows to determine the optimal coefficients ACS CPU considering engineering criteria for the quality of ACS.

At the first stage, the values of $\mathrm{K}_{P}$, and $\mathrm{K}_{D}$ are determined, which provide the maximum value of static coefficient of transmission systems with a monotonic nature of the transition processes. In the second stage, the coefficient of the integrator $\mathrm{K}_{I}$ is determined, providing the highest system performance in the class of systems with monotonic transition processes
Minimizing the time of the transition process in the class of systems with monotonic processes is provided by the proposed definition of a minimum criterion of optimization. Then, the output value of the integrator is limited.

After finding the optimal coefficients for all the calculated modes of CPU according to [6], the coefficients $\mathrm{K}_{P}, \mathrm{~K}_{D}$ and $\mathrm{K}_{I}$ are converted into the functional dependence KP (), KD () and $\mathrm{KI}$ () on the mode of CPU (as a mode of operation is the Frequency of rotation of the CPU drive).

For example, the coefficient of differentiation of the functional dependence of the frequency of rotation of the CPU drive will look like:

$$
K_{P}(\varphi)=0,3685 \varphi^{2}-1,4105 \varphi+9,6641
$$

\section{THE SIMULATION RESULTS}

Experimental study of optimal digital ACS CPU, tailored to the characteristics, carried out by simulation in the Matlab 6.5. Studies were included to obtain the transition processes in the system mode $\mathrm{p}=100 \%$ due to a step change of control action on the power supplied to the drive CPU. The results are shown in the table.

TABLE I. Simulation Results

\begin{tabular}{|c|c|c|c|}
\hline $\begin{array}{c}K_{P}=70,2 \\
K_{D}=8,6\end{array}$ & $\Delta \varphi$ & $\Delta L$ & $\begin{array}{c}\text { transition process } \\
\text { time }\end{array}$ \\
\hline$K_{I}=0$ & $83,6^{\circ}$ & $32,7 \mathrm{~dB}$ & $2 \mathrm{c}$ \\
\hline$K_{I}=103,8$ & $81,8^{\circ}$ & $32,7 \mathrm{~dB}$ & $1 \mathrm{c}$ \\
\hline
\end{tabular}

In the first stage Under certain optimal coefficients $K_{P}=$ 70,2 and $K_{D}=8,6$ and off the integrator transition processes time is 2 seconds. After a start operation of the integrator the found optimal value of the coefficient of integration stability margin in phase $\Delta \varphi$ decreased to 1.6 , and the transition process time was reduced to 1 second.

Transition processes have monotonous periodic character. Stability margins optimal system developed to meet the considered system features a 3 times higher than specified in the design of quality criteria for $\Delta \varphi \geq 45^{\circ}, \Delta L \geq 12 \mathrm{~dB}$.

\section{CONCLUSIONS AND SUGGESTIONS}

This paper has proposed a method of designing a digital system that takes into account the CPU main features as a control object and the requirements of the regulatory system.

It is advisable on this basis to develop software that allows in the operation of the pump unit automatically determine the optimal parameters of the control system taking into account the system requirements and characteristics of the controlled object.

\section{REFERENCES}


[1] Don Morrison. Is it time to replace PID? Applying model-predictive control at the single-loop level may yield the next big leap in performance gains. URL: http://www.isa.org // 01 March 2005

[2] PID controller: From Wikipedia, the free encyclopedia URL:http://en.wikipedia.org/wiki/PID_controller.

[3] Minakov A.V., Belousov, G.G. Optimal software implementation of digital PID regulators with feedback for objects with low response time and transition characteristics of high-order. // Automation, telemechanization and communication in the oil industry. - Moscow: JSC "VNIIOENG", 2008. - № 4: URL:http://vniioeng.men.ru/inform/avtomatisation/sod4.

[4] Dorf, R. Bishop, R. Modern control systems. - Moscow: Laboratory of Basic Knowledge, 2002. URL: http://www.twirpx.com/file/21901/

[5] Kuznetsova E.E. The method of designing digital pressure regulator at the output of the pump unit / / Automation in industry. - 2009. - № 4. URL: http://avtprom.ru/\%C2\%A0-metodika-proektirovaniya-tsifrovogore.
[6] Kuznetsova E.E., Lyantsev O.D., The method of determining the coefficients of PID regulator pump unit // Automation, telemechanization and communication in the oil industry. - Moscow: JSC "VNIIOENG", 2010 .

№

4.

URL: http://www.nefteavtomatika.ru/Publication127_13_159.aspx.

\section{AUTHOR'S PROFILE}

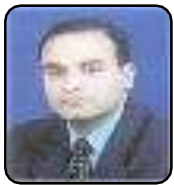

Dr. Osama Ahmad Salim Safarini had finished his PhD from The Russian State University of Oil and Gaz Named after J. M. Gudkin, Moscow, 2000, at a ComputerizedControl Systems Department.

$\mathrm{He}$ obtained his BSC and MSC in Engineering and Computing Science from Odessa Polytechnic National State University in Ukraine 1996. He worked in different universities and countries. His research is concentrated on Automation in different branches. 